[Vicino Oriente XVIII (2014), pp. 25-38]

\title{
MAIKI, MISSIONE ARCHEOLOGICA ITALIANA NEL KURDISTAN IRACHENO: LA CARTA ARCHEOLOGICA DELL'AREA DI PAIKULI, OBIETTIVI E METODOLOGIE APPLICATE
}

\author{
Kamal Rashid Rahim - Direttorato delle Antichità di Sulaimaniyah \\ Carlo G. Cereti - Luca Colliva - Agnese Fusaro - Camilla Insom - Giuseppe Labisi - \\ Serenella Mancini - Gianfilippo Terribili - Sapienza, Università di Roma \\ Julian Bogdani - Marco Galuppi - Ass. ROUTES
}

\begin{abstract}
In the fall of 2012 the Italian Archaeological Mission in the Iraqi Kurdistan signed an agreement with the Sulaimaniyah Antiquities Directorate, for creating the Archaeological Map of the area surrounding the Sasanian monument of Paikuli. In the course of the four campaigns, which have been carried out, the team has started to survey the area, has developed a methodology for the classification of ceramic materials and started the preliminary study of the findings. Furthermore, in 2013 MAIKI commenced an ethnographic research aimed at studying the religious heritage of the area.
\end{abstract}

Keywords: Kurdistan; Paikuli; archaeological map; pottery; sheykh

La Missione Archeologica Italiana nel Kurdistan Iracheno (MAIKI) del Dipartimento di Scienze dell'Antichità della "Sapienza" Università di Roma, condiretta dalla Prof.ssa Maria Vittoria Fontana e dal Dr. Luca Colliva, si propone lo studio dello sviluppo storico, linguistico e culturale della regione curda in epoca storica. Dalla sua nascita nel 2011, quale erede delle attività del team italiano guidato dal Prof. Carlo Giovanni Cereti e attivo nel Kurdistan Iracheno dal $2006^{1}$, la MAIKI ha avviato una proficua collaborazione con gli enti locali curdi deputati allo studio e alla salvaguardia dei beni storico-archeologici e culturali della regione e in particolare con i Direttorati delle Antichità, con l'High Commission for the Erbil Citadel Revitalization (HCECR) e con i tre principali musei archeologici della regione, il Museo di Sulaimaniyah, il Museo di Erbil ed il Museo di Duhok. Tutte le attività vengono programmate e realizzate in sinergia con questi enti locali, prevedono la partecipazione attiva di archeologi e funzionari curdi e sono effettuate grazie al sostegno del Ministero degli Affari Esteri e della Cooperazione Internazionale, dell'Ambasciata d'Italia a Baghdad e dell'Ufficio Consolare Italiano a Erbil.

In questi anni la MAIKI ha scelto di focalizzare le sue attività sullo studio del sito archeologico di Paikuli e dell'area ad esso limitrofa (fig. 1). Il monumento sasanide di Paikuli sorge sui primi contrafforti occidentali dei Monti Zagros in prossimità di uno dei passi che permettono l'attraversamento di questi rilievi montuosi; nell'antichità questa zona si collocava al confine tra la provincia sasanide nota come Āsūrestān, la provincia reale dov'era situata la capitale Ctesifonte, e le regioni dell'altopiano iranico; in questo luogo i notabili dell'impero attesero l'arrivo di Narseh (293-302 d.C.) dopo la sua vittoria su Wahrām III per riconoscerlo come nuovo sovrano dell'impero sasanide. A memoria di questo avvenimento il sovrano fece erigere un imponente monumento turriforme

1 Cereti - Giunta 2011; Cereti et al. 2012. 
caratterizzato dalla presenza di una lunga iscrizione bilingue in mediopersiano e partico, nella quale vengono narrate le vicende che lo portarono al trono (fig. 2).

Nell'autunno del 2012 la MAIKI ha raggiunto un accordo quinquennale con il Direttorato delle Antichità di Sulaimaniyah, guidato dal Dr. Kamal Rashid Rahim, per lo studio del monumento di Paikuli e della sua iscrizione e per la realizzazione di una carta archeologica dell'area. Agli studi storico-filologici sull'iscrizione bilingue di Narseh, portati avanti dal Prof. C.G. Cereti e dal Dr. Gianfilippo Terribili fin dal 2006 e ora confluiti nella $\mathrm{MAIKI}^{2}$, si sono pertanto affiancate attività di survey nonché di studio storico-archeologico e antropologico del territorio.

\section{LA CARTA ARCHEOLOGICA DELL'AREA DI PAIKULI}

Il programma della MAIKI prevede la realizzazione di una carta archeologica della valle lungo la quale corre la strada che collega le città di Chamchamal e Darbandikhan; l'area, delimitata a nord e a sud dai fiumi Basra e Dyala, copre una superficie di circa 835 $\mathrm{km}^{2}$ e comprende gran parte dei sottodistretti di Sangaw e Pebaz (fig. 1$)^{3}$.

La zona è stata oggetto di survey pubblicato nella seconda metà del secolo scorso; da allora, complici l'instabilità politica e gli scontri militari, le attività archeologiche nella regione sono state minime, se non completamente assenti.

Date queste premesse, la realizzazione di una carta archeologica aggiornata dell'area, che permetta l'identificazione e il posizionamento di tutti i siti archeologici o di interesse storico-culturale, rappresenta non solo uno strumento fondamentale per lo studio dell'occupazione antropica della regione, ma anche un elemento propedeutico per ogni attività di protezione e valorizzazione dei beni storico-archeologici presenti nell'area. La consapevolezza dei rischi che corre il patrimonio storico-archeologico della regione, a causa della repentina crescita economica ed edilizia prima e dello scontro in atto con Daesh ora, ha reso prioritaria la necessità, per i Direttorati delle Antichità, di dotarsi con urgenza di tutti gli strumenti atti a proteggere questo immenso patrimonio.

Questi pericoli sono presenti anche nell'area oggetto del nostro studio, nonostante essa sia scarsamente abitata e solo marginalmente coinvolta dal repentino sviluppo economico della regione. Due siti archeologici sono stati distrutti e altrettanti sono stati danneggiati da opere di livellamento e irrigazione connesse alla crescente attività agricola. Nel corso delle due campagne di survey, inoltre, in almeno due occasioni sono state trovate, e immediatamente segnalate alle autorità competenti, tracce di scavi clandestini.

Le attività legate alla realizzazione della carta archeologica hanno avuto inizio nel 2012 Ad oggi la MAIKI ha realizzato quattro campagne, due dedicate al survey e alla raccolta di dati sul territorio e due focalizzate sullo studio e la catalogazione dei materiali archeologici raccolti ${ }^{4}$.

\footnotetext{
Cereti - Terribili 2012; 2014
}

I confini amministrativi dell'area sono attualmente soggetti a frequenti modifiche; per i nomi e i confini dei sottodistretti sono state usate come riferimento le mappe realizzate nel 2001 da UNOPS e UNJHIC.

4 Le quattro campagne hanno visto la partecipazione, insieme al team italiano e sotto la costante supervisione del loro direttore, il Dr. Kamal Rashid Rahim, di Zana Abdullkarim Qadir, Nawshirwan Aziz Mohammed, Khanda Osman Fatah e Parwin Yawar Manda del Direttorato delle Antichità di Sulaimaniyah. 
La ricognizione sul terreno è stata preceduta da uno studio preliminare di immagini satellitari di diversa provenienza e data. Tra queste si distinguono le immagini CORONA, recentemente declassificate, precedenti quello che è sicuramente l'evento recente di più grande impatto sul popolamento della regione, ovvero la campagna di al-Anfal (1986-1989) condotta da Saddam Hussein contro la popolazione curda (v. infra). Uno strumento fondamentale, inoltre, è costituito dalla banca dati geografica fornita da Google (Google Earth) che offre immagini attuali a buona definizione per gran parte dell'area sotto esame. Una terza fonte è costituita da stereo-coppie di immagini QuickBird ad alta risoluzione, acquistate per porzioni limitate del territorio (in particolare l'area nelle immediate vicinanze di Paikuli). L'analisi di queste fonti ha portato all'individuazione di quasi duecento possibili siti di interesse archeologico e antropologico. Questo tipo di analisi, però, non può che essere preliminare a una ricognizione sul campo. Le primissime campagne, infatti, hanno chiarito molte situazioni dubbie osservate dalle immagini satellitari: in diversi casi piccoli villaggi abbandonati possono essere facilmente interpretabili, a prima vista, come potenziali aree archeologiche e solo una verifica sul campo è in grado di stabilire in modo chiaro la loro natura. D'altro canto diverse tipologie di siti archeologici, facilmente individuabili dalle ricognizioni sul campo, non lasciano sul terreno tracce osservabili dall'alto ${ }^{5}$. Le attività di survey si sono focalizzate, in questa primissima fase, sulla verifica dei siti segnalati nell'atlante iracheno edito nel 1975 e delle aree a essi limitrofe. Trattandosi, nella quasi totalità dei casi accertati, di siti di dimensioni importanti è chiaro come una loro adeguata e aggiornata documentazione sia premessa necessaria a qualsiasi ricostruzione storica del sistema insediativo dell'area in esame. La ricognizione intensiva dell'intero territorio è in atto, pur con evidenti limiti dovuti ai recenti conflitti e al fatto che non tutta la zona interessata dal survey sia stata bonificata dalla presenza di mine antiuomo.

Un ulteriore problema che si è riscontrato fin dalle prime fasi di lavoro è quello della mancanza sostanziale di una cartografia di base adeguata. A questa si aggiunge la situazione caotica creatasi dopo i conflitti della fine degli anni ' 80 del secolo scorso con i toponimi dei villaggi. Durante la campagna di al-Anfal tutti i centri dell'area sotto esame furono spopolati e distrutti. In seguito alla Prima Guerra del Golfo molti centri sono stati ricostruiti nello stesso sito dei precedenti, mentre altri sono stati riedificati in luoghi differenti, pur mantenendo il toponimo d'origine. Infine, nel corso di questi ultimi 25 anni sono stati costruiti nuovi insediamenti, spesso di piccole o piccolissime dimensioni, che riprendono anch'essi il toponimo utilizzato per indicare il villaggio principale dell'area. L'attuale modello insediativo, dunque, è quantomeno dinamico e non ancora cristallizzato, sia per quanto riguarda posizioni e dimensioni dei centri sia per quel che riguarda la toponomastica. Ė interessante osservare come gli abitanti dell'area, per la maggior parte sedentarizzati da non più di 100-120 anni, non percepiscano questa confusione nella toponomastica e nella stessa dislocazione spaziale dei villaggi come un'anomalia ${ }^{6}$. Per formalizzare al meglio la situazione fin qui descritta si è resa necessaria una mappatura di

5 Durante le ricognizioni sono state rilevate due tombe rupestri (MPS-S0013A, MPS-S0014) e due aree con dispersione di materiale litico (MPS-S0009; MPS-S0010) non visibili nelle foto satellitari.

6 Vi sono numerose testimonianze del fatto che l'apposizione di più nomi a un villaggio sia uso comune nelle aree rurali del Kurdistan iracheno. 
tutti i centri attualmente abitati nell'area, segnandone la posizione precisa e il toponimo usato dalle persone che vi abitavano, in modo da realizzare ex novo la cartografia di base, strumento fondamentale anche per la programmazione e il proseguimento della ricerca antropologica.

Tutti questi dati sono stati inseriti in una piattaforma GIS collegata a un database relazionale collaborativo creato allo scopo, che raccoglie tutti i dati geografici acquisiti, le informazioni di carattere prettamente archeologico e i dati antropologici raccolti ${ }^{7}$.

Le due campagne di survey fino ad ora realizzate hanno portato al riconoscimento di 95 villaggi, 30 siti archeologici, 21 cimiteri moderni o contemporanei e 17 aree legate al culto degli sheykh (vedi infra). Tutti i luoghi di interesse, identificati con l'acronimo MSP (MAIKI Survey Project) seguito da un numero progressivo di quattro cifre, sono stati georeferenziati grazie all'utilizzo di un GPS palmare e inseriti nel database e nella piattaforma GIS (figg. 3-4).

Per ogni sito archeologico è stata indicata la presunta area di estensione, riconosciuta attraverso la presenza di strutture affioranti o materiali di superficie, e l'eventuale presenza di sotto-unità (complex $)^{8}$, nei casi in cui erano evidenti aree diverse per funzione, tipologia dei resti o cronologia dei manufatti archeologici. La presunta area di estensione di ogni complex è stata anch'essa annotata.

In ogni sito è stato raccolto tutto il materiale ceramico considerato diagnostico e tutti i tipi d'impasto attestati. Tutti i materiali litici e gli oggetti metallici non contemporanei (ovvero non ascrivibili al XX e XXI secolo) sono stati raccolti; le ossa sono state raccolte solo quando rinvenute in contesti archeologici significativi (tombe, stretta connessione con altri materiali archeologici, ecc.) o in caso di presenza di tracce di lavorazione.

Lo studio dei siti e dei manufatti archeologici è ancora in fase preliminare e i dati di cui disponiamo sono da considerare estremamente parziali; tuttavia possiamo confermare un'occupazione antropica dell'area almeno dal periodo protostorico e l'esistenza di siti di grandi dimensioni che presentano tracce di un insediamento continuato quanto meno dal periodo di Uruk a quello proto-islamico (MPS-S0013).

Una prima analisi dei materiali, ed in particolare il ritrovamento di frammenti di Bevelled Rim Bowls, ha permesso di identificare fasi relative al periodo di Uruk in due siti (MSP-S0013 e MSP-S0034); quattro siti (MSP-S0005, MSP-S0012, MPS-S0013 e MSPS0031) presentano materiali databili al periodo ellenistico, Bowls with Inturned Rim, e due siti (MSP-S0013 e MSP-S0037) presentano frammenti di ceramica decorata a stampo con iconografie sasanidi (Fig. 5). Ceramica proto-islamica (VIII secolo) e di periodo abbaside (VIII-XIII secolo) è stata rinvenuta in cinque siti (MSP-S0004, MSP-S0012, MSP-S0013 MSP-S0032 e MSP-S0037; fig. 6), mentre manufatti di periodo ottomano (XVI-XVIII secolo), come le caratteristiche Ottoman Pipes, sono attestati in sette dei siti visitati (MSPS0004, MSP-S0008, MSP-S0011, MSP-S0012, MSP- S0013, MSP-S0033 e MSP-S0045).

7 Si ringrazia la BraDypUS s.a., l'ass. culturale Routes e lo Studio 3R per il supporto fornito alla MAIKI attraverso la realizzazione, lo sviluppo e il costante aggiornamento degli strumenti informatici della missione.

8 I complex sono indicati dal codice identificativo del sito seguito da una lettera maiuscola (es. MPS-S0013A). 


\section{LO STUDIO DEI MATERIALI ARCHEOLOGICI}

I materiali raccolti nel corso del survey costituiscono un corpus quantitativamente notevole e molto eterogeneo; ogni classe di materiali, inoltre, presenta una vasta gamma di produzioni, relative a un ampio arco cronologico.

Durante le due campagne dedicate allo studio dei reperti è stata messa a punto la metodologia di classificazione dei materiali ed è stato avviato lo studio preliminare dei ritrovamenti. Tra i materiali studiati particolare attenzione è stata dedicata alla ceramica.

La classificazione è finalizzata all'identificazione delle differenti produzioni attestate nell'area, al riconoscimento del livello tecnologico raggiunto dagli artigiani locali nei diversi periodi di occupazione e all'individuazione dei manufatti di importazione e della loro provenienza. Questi studi sono funzionali alla datazione dei siti archeologici e al riconoscimento dell'orizzonte cronologico delle differenti fasi di occupazione dell'area. Lo studio dei materiali si avvale di analisi archeometriche realizzate in collaborazione con il Prof. Mario Piacentini del Dipartimento di Scienze di Base e Applicate per l'Ingegneria della Sapienza Università di Roma e con il Dr. Giolj Guidi. I dati sui materiali confluiscono anch'essi nel database relazionale utilizzato per i siti archeologici e a questo fine sono state messe a punto tre schede attinenti ai corpi ceramici identificati (Fabric), ai raggruppamenti ceramici riconosciuti (Group) e agli oggetti (Object).

Nella scheda Object sono elencati tutti gli attributi che descrivono il manufatto selezionato: oltre alle informazioni relative alla provenienza e agli aspetti tecnologici, morfologici e stilistici, si propone anche un'attribuzione cronologica e si annotano pubblicazioni utili allo studio comparativo del manufatto.

Per quanto concerne la ceramica, la varietà delle produzioni attestate e il fatto che si tratti di materiale proveniente da ricognizione e, di conseguenza, non databile sulla base del contesto archeologico, hanno imposto la scelta di un sistema di classificazione che sia duttile e applicabile a qualsiasi categoria ceramica. In questa prima fase si è reso necessario tenere in considerazione tutti gli aspetti tassonomici, dimensionali e qualitativi al fine di ottenere una descrizione il più possibile completa dei frammenti in esame. Per questo motivo si è deciso di combinare in un unico sistema due metodologie di lavoro, associate a tradizioni di studi che valorizzano caratteristiche differenti: una che pone l'accento sulla forma (tassonomia morfo-dimensionale) e un'altra che privilegia l'analisi del corpo ceramico e del trattamento della superficie in associazione alla forma.

Cardine di questo sistema di classificazione è il concetto di raggruppamento ceramico (Group): con questo termine s'identifica l'insieme delle principali caratteristiche o attributi che descrivono in maniera dettagliata un gruppo di frammenti simili. Tali caratteristiche sono: corpo ceramico, tecnica di modellazione, trattamenti superficiali, spessore delle pareti $^{9}$.

9 La definizione di raggruppamento ceramico si avvicina al concetto di ware come impiegato nell'archeologia americana (Klejn 1982, 52) e come inteso nel 'type-variety system' (Rice 1987, 286-287; Sinopoli 1991, 5253 ); tuttavia si è evitato di impiegare il termine ware, dal momento che nella letteratura specialistica esso indica generalmente una produzione ceramica ben definita, mentre i raggruppamenti ceramici descritti in questa fase possono essere considerati come varianti interne delle produzioni ceramiche. 
Per facilitare e velocizzare l'inserimento dei dati e rendere intuitiva la consultazione delle informazioni si è scelto di denominare i raggruppamenti ceramici attraverso un codice alfanumerico, rappresentativo degli attributi identificati: ad esempio C001-WH-POL-F. La prima sigla corrisponde al numero del corpo ceramico, la lettera iniziale fa riferimento alla natura della matrice: argillosa, silicea, etc. (nel caso dell'esempio $\mathrm{C}$ indica un corpo argilloso, $\mathrm{C}=$ Clay). Il corpo ceramico (Fabric) è il primo degli attributi esaminati al momento dell'analisi. I caratteri considerati nell'analisi di ciascun corpo ceramico sono: colore, porosità, durezza e compattezza e l'aspetto complessivo del corpo ceramico. Infine si elencano gli inclusi vegetali e minerali di cui si valutano la frequenza, le dimensioni, il grado di arrotondamento.

La seconda sigla del codice, che identifica il Group, indica la tecnica di modellazione (nell'esempio presentato è stato riconosciuto l'uso del tornio, $\mathrm{WH}=W H e e l$ ); seguono una o più sigle relative ai trattamenti di superficie, ovvero le tecniche di rifinitura della superficie, le tecniche decorative e i rivestimenti (nell'esempio il frammento presenta una superficie lucidata, POL $=$ POLished). L'ultima sigla corrisponde allo spessore del frammento, indicatore dell'aspetto morfo-dimensionale del manufatto ceramico (nell'esempio $\mathrm{F}$ è la sigla che indica una ceramica fine $)^{10}$.

All'interno del codice del raggruppamento ceramico non rientra l'indicazione della forma, dal momento che a un raggruppamento ceramico possono corrispondere forme diverse che andranno a formare una serie di sotto-gruppi interni del raggruppamento ceramico. Per quel che riguarda lo studio delle forme è in corso di elaborazione una classificazione morfologica che ricorra anch'essa ad un codice alfanumerico; questa scelta è stata effettuata non solo per velocizzare la rappresentazione e la consultazione, ma soprattutto al fine di ottenere una descrizione delle forme quanto più possibile oggettiva, evitando l'uso di terminologie ambigue.

Limitatamente allo studio del materiale ceramico, la scheda Object è utilizzata per schedare forme complete o frammenti selezionati. Sono stati selezionati tutti i frammenti che per forma, particolarità tecnologiche (relative alle tecniche di modellazione o ai trattamenti della superficie), tecniche e motivi decorativi sono da considerare significativi per l'identificazione delle produzioni ceramiche attestate nell'area e rappresentativi dell'intero corpus.

\section{LA RICERCA ETNOGRAFICA}

Nell'intenzione di ricostruire il peculiare contesto storico-culturale della valle per la quale è in atto la stesura della carta archeologia, la MAIKI ha avviato un programma di ricerca etnografica il cui scopo primario è lo studio del patrimonio religioso e delle pratiche cultuali ad esso legate. Le attività di ricerca sul campo sono iniziate nel dicembre 2013 e a oggi sono state compiute due campagne ricognitive volte a chiarire e individuare l'oggetto di ricerca che costituirà il fulcro del progetto etnografico.

10 La ceramica è stata suddivisa sulla base degli intervalli degli spessori in tre gruppi: fine (spessore inferiore a $0,5 \mathrm{~cm}$ ), medium (spessore tra 0,5 e $1,5 \mathrm{~cm}$ ) e thick (spessore superiore a $1,5 \mathrm{~cm}$ ). 
I dati raccolti, benché parziali e riferibili solo a una piccola sezione del territorio in esame, indicano la capillare presenza di luoghi di culto dedicati agli sheykh, maestri spirituali delle confraternite sufi.

Nell'area a oggi indagata, dove insistono 30 villaggi, di cui 20 abitati, sono stati individuati 17 santuari di sheykh: 13 tombe all'interno di cimiteri, 1 tomba all'interno di una moschea, 2 luoghi di eremitaggio e 1 un mausoleo, nella città di Krpchna, sede della Kasnazaniyya, una branca della Qādiriyya, la più antica delle principali confraternite sufi oggi presenti nel Kurdistan iracheno (figg. 7-8).

La diffusione dei santuari sul territorio, unita alle testimonianze raccolte sul campo e alla letteratura di riferimento ${ }^{11}$, permette di delineare $\mathrm{i}$ contorni di un sistema cultuale legato alla venerazione degli sheykh. La popolazione locale, di credo sunnita, reputa gli sheykh santi, in netto contrasto con lo spiccato teocentrismo dell'Islam ortodosso, e li riveste del ruolo d'imprescindibili intermediari con la divinità. Il potere degli sheykh si manifesta nel mondo come capacità di compiere azioni taumaturgiche e divinatorie. Tale potere non si esaurisce alla loro morte. Rituali terapeutici, apotropaici e propiziatori, che spesso includono il sacrificio animale, sono posti in atto laddove il potere degli sheykh permane oltre la morte, in particolar modo dunque sulle tombe.

Il culto degli sheykh, attuale fino all'abbandono della valle nel $1988^{12}$ a seguito della terza campagna di al-Anfal, pare oggi interessato da un profondo quanto repentino mutamento. Nonostante la venerazione degli sheykh permanga viva nella memoria collettiva e vi siano, sul territorio, soggetti popolarmente riconosciuti come santi ed eredi dell'antico ruolo religioso e sociale detenuto dai loro predecessori, l'attualità del culto appare problematica.

Nel corso delle interviste l'intero sistema cultuale, e in particolare la ritualità sacrificale, è affermato solo quando riferito a un passato anteriore la deportazione. Viceversa, interrogati in riferimento al presente, la quasi totalità degli informatori afferma di non mettere più in atto alcuna azione rituale legata al potere degli sheykh, ma è altresì in grado di indicare chi ancora lo fa. Nella maggior parte dei casi sono le donne, primariamente anziane, a riferire di compiere tuttora riti terapeutici o divinatori ad essa legati, mentre tra gli uomini si riscontra una marcata resistenza a trattare l'argomento e non sono rare le affermazioni di viva condanna nei confronti di pratiche indicate come frutto d'ignoranza. In generale laddove si registra il permanere della frequentazione dei santuari degli sheykh, si rileva un'epurazione delle componenti taumaturgiche della ritualità praticata, nel segno di un reinserimento nel quadro dell'Islam ortodosso: i santuari vengono così reinterpretati quali luoghi di semplice riverenza.

Benché la ricerca sia nella sua fase germinale è possibile identificare alcuni dei fattori che possono aver concorso a tale modificazione:

11 La devozione per i santi sufi non è una peculiarità della regione ma fattore che accomuna ampie zone del mondo islamico. Per una panoramica attuale si veda Werbner - Bosu 1998; per il contesto curdo si veda van Bruinessen 1992, 210-219.

12 Sulla base delle testimonianze raccolte sul campo è possibile affermare che l'abbandono della valle rappresenti un momento di cesura radicale per il sistema religioso locale. 
- la migrazione forzata, il distacco fisico dal territorio, e la conseguente rescissione dei legami tra gli sheykh e la popolazione;

- l'inurbamento coatto della popolazione rurale, che nel 1988 ha posto gli abitanti dei villaggi a contatto con istituzioni estranee al sistema tradizionale;

- l'influenza degli imam, che dal 1991, nel corso della predicazione del venerdì, hanno iniziato a negare apertamente la realtà del ruolo intercessorio con la divinità degli sheykh; a questo proposito è interessante osservare come la valle abbia visto l'attuarsi di un programma, finanziato da organizzazioni filantropiche saudite, volto alla ricostruzione delle moschee presenti nei villaggi ${ }^{13}$;

- l'indebolimento dell'autorità religiosa degli sheykh, il cui potere pare oggi esercitarsi primariamente, se non esclusivamente, nella sfera politica. Da ciò consegue il progressivo abbandono dell'attributo di 'santità', all'origine del potere taumaturgico e divinatorio.

La ricerca etnografica si propone di indagare il mutamento in atto nel sistema cultuale connesso alla venerazione degli sheykh. Ci si attende di delineare un quadro esaustivo, per l'area in esame, dei riti terapeutici, apotropaici o propiziatori legati alla venerazione degli sheykh, così come messi in atto nel periodo immediatamente precedente al-Anfal, di tracciare l'attualità delle pratiche rituali in questione e di analizzare i possibili fattori che concorrono nel guidare il mutamento in atto.

Per quanto attiene la metodologia ci si propone di suddividere la ricerca in tre fasi. L'assenza di documentazione etnografica ${ }^{14}$ e demografica, come di cartografia relativa al territorio, rende necessario impostare una prima fase, ora in atto, sulla raccolta dei dati propedeutici al successivo sviluppo della ricerca. Si è deciso dunque di completare la mappatura dei villaggi, dei cimiteri, dei luoghi di rilevanza cultuale e di raccogliere sul campo, mediante l'identificazione di un informatore in ogni villaggio, dati quantitativi e qualitativi circa la composizione demografica e i movimenti della popolazione, con particolare riguardo per le modificazioni riscontrabili successivamente all'Anfal, i raggruppamenti genealogici, le strategie matrimoniali, l'economia e le pratiche religiose. In questa fase si procederà, inoltre, alla raccolta di testimonianze sui riti terapeutici, apotropaici e propiziatori legati alla venerazione degli sheykh. Laddove presenti, si raccoglieranno informazioni circa l'agiografia dello sheykh cui il luogo è legato, i peculiari effetti del suo potere e le azioni rituali messe in atto al fine di suscitarlo. La citata ritrosia riscontrata nella popolazione locale a trattare l'argomento suggerisce di focalizzare le

13 Con imam indichiamo qui la guida della preghiera sunnita, perché la popolazione della valle, così come quella dell'intero Kurdistan, è prevalentemente di credo sunnita e non sciita.

14 La letteratura antropologica relativa al Kurdistan iracheno è esigua. Per quanto attiene l'organizzazione sociale e politica, permangono centrali le ricerche compiute da Edmund Leach nelle province di Sulaymaniyah e Kirkuk (Leach 1940) e Fredrik Barth nella provincia di Erbil (Barth 1953). La peculiare complessità del panorama religioso curdo è stata studiata con particolare riguardo sotto il profilo storico religioso (Kreyenbroek - Allison 1996) primariamente per quanto attiene le sette sincretiche degli Yazidi (Kreyenbroek 1995) e degli Ahl-e aqq (van Bruinessen 1995) e le congregazioni sufi della Qādiriyya e Naqshbandiyya (Gaborieau - Popovic - Zarcone 1990; van Bruinessen 1999); particolarmente rilevante per la ricerca che qui si presenta è l'analisi socio politica del ruolo degli sheykh del Kurdistan compiuta da Martin van Bruinessen sulla base di una ricerca condotta in Iran, Iraq e Turchia all'inizio degli anni ' 70 (van Bruinnesen 1992) 
interviste, di questa prima fase, sulla pratica rituale del periodo precedente al-Anfal e solo successivamente, a seguito di un più prolungato periodo di permanenza sul campo, verificarne l'attualità.

Si prevede di focalizzare la seconda fase del progetto sullo studio delle pratiche rituali nel momento presente, sottoponendo le conoscenze acquisite a un campione etnografico che tenga contro delle fasce d'età come della stratificazione sociale. In questa successiva fase ci si propone inoltre lo studio dei fattori che, sulla base delle indicazioni precedentemente raccolte, s'ipotizza possano aver concorso nell'ingenerare il mutamento della pratica rituale. Verranno pertanto raccolte testimonianze relative al trasferimento forzato nelle città nel periodo dal 1988 al 1991, alla predicazione degli imam, e si tenterà di reperire in loco fonti documentali e d'informazione relative al programma di ricostruzione delle moschee nella valle finanziato dalle organizzazione filantropiche saudite. Le fonti d'informazione e documentali, unite alla relativa letteratura di riferimento e alle testimonianze raccolte sul campo, forniranno la base per l'analisi della supposta traslazione del campo d'autorità degli sheykh dalla sfera religiosa all'arena politica.

Da ultimo s'ipotizza di allargare il terreno di ricerca alla città di Sulaymaniyah, sede da alcuni anni del centro del lignaggio della Qādiriyya di Krphona, al fine di considerare le possibili differenze nelle pratiche rituali legate alla venerazione degli sheykh nell'ordito sociale proprio del contesto urbano, rispetto all'area rurale.

\section{BIBLIOGRAFIA}

BARTH, F.

1953 Principles of social organization in southern Kurdistan, Oslo 1953.

VAN BRUINESSEN, M.

1992 Agha, Shaikh, and State: the social and political structures of Kurdistan, London 1992.

1998 The Kurds and Islam: Les annales de l'autre islam 5 (1998), pp. 13-35.

1999 The Qadiriyya and the lineages of Qadiri shaykhs in Kurdistan: Journal of the History of Sufism 1 (1999), pp. 213-229.

CERETi, C.G. - GiUnTA, R. (eds.)

2011 Preservation of Cultural Heritage of the Kurdish Region in Iraq, Bologna 2011.

Cereti, C.G. - Colliva, L. - Fontana, M.V. - Terribili, G. - Bogdani, J. - Bizzarro, A. - Tilia, A. TILIA, S.S.

2012 From Flint to Silicon, Modern Technologies Applied to the Understanding of History. The Italian Archaeological Mission in Iraqi Kudistan: Vicino Oriente XVI, pp. 181-200.

Cereti, C.G. - TerribiLi, G.

2012 The Paikuli Monument: M. Alram - R. Gyselen (eds.), Sylloge Nummorum Sasanidarum, Band II, Wien 2012, pp. 74-87.

2014 The Middle Persian and Parthian Inscriptions on the Paikuli Tower. New Blocks and Preliminary Studies: Iranica Antiqua 49 (2014), pp. 347-412.

GABORIEAU, M. - Popovic, A. - ZARCONE, TH.

1990 Naqshbandis historical development and present situation of a Muslim mystical order: proceedings of the Sevres round table 2-4 May 1985. Istanbul and Paris 1990.

HUMAN RighTS WATCH,

1995 Iraq's crime of genocide: the Anfal campaign against the Kurds. New Haven 1995. 


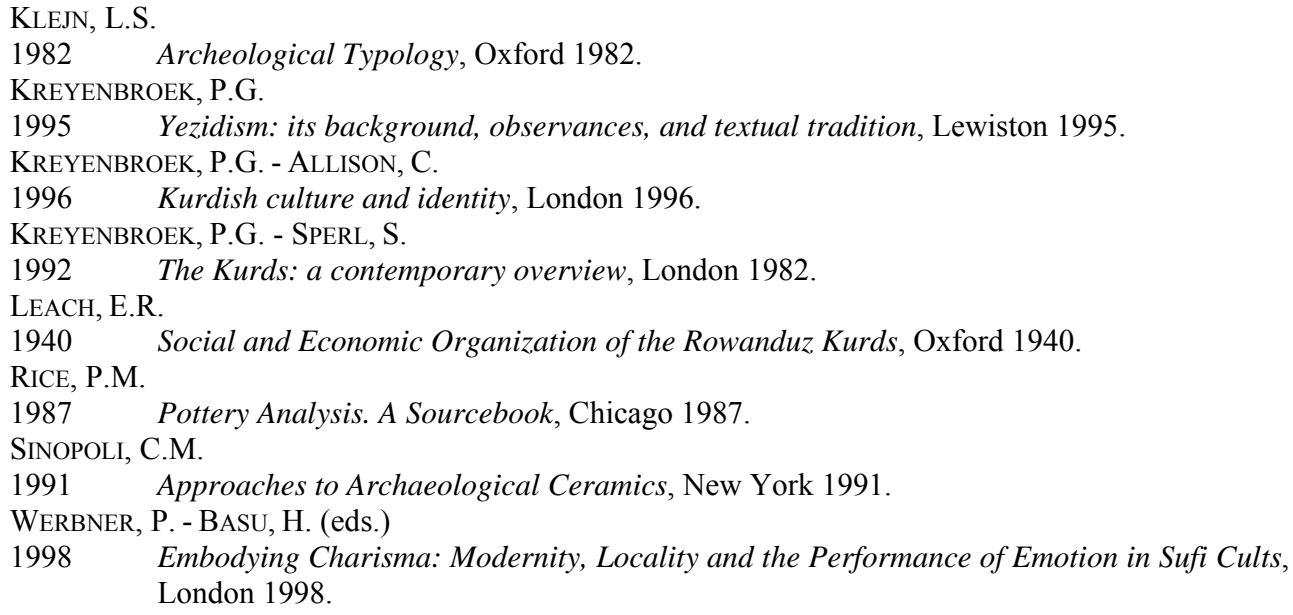




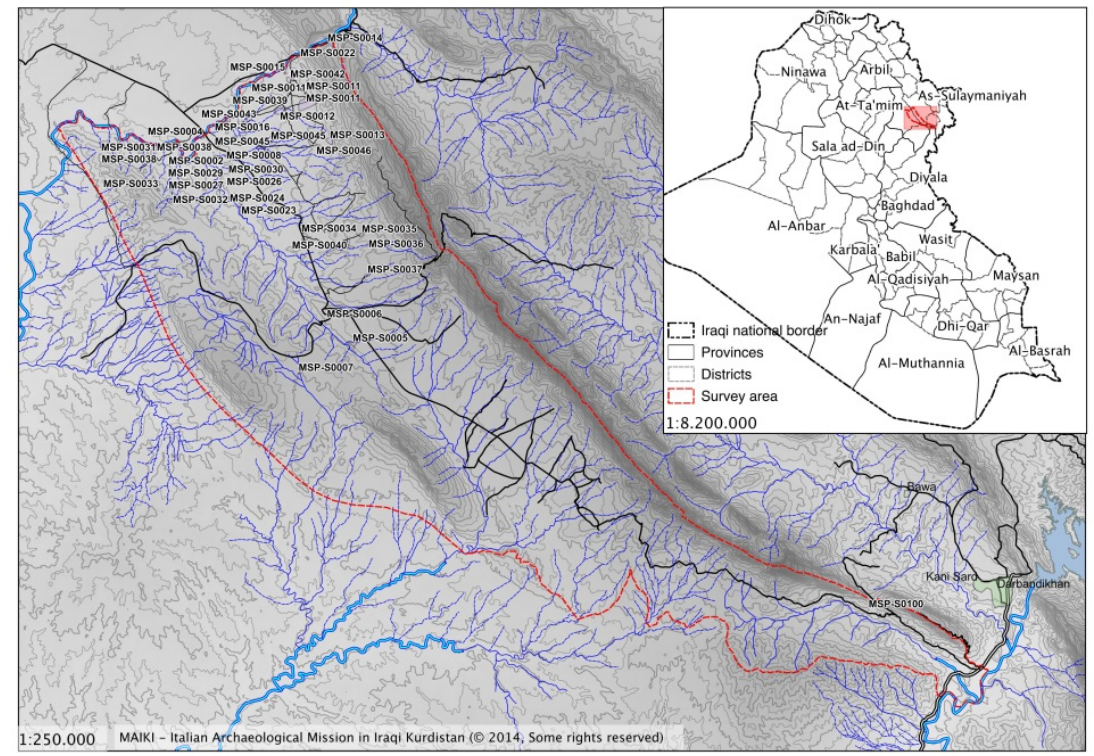

Fig. 1 - Iraq; l'area di indagine del MAIKI Survey Project. Il sito di Paikuli si trova nell'angolo SE della mappa (MSP-S0100) (immagine MAIKI).

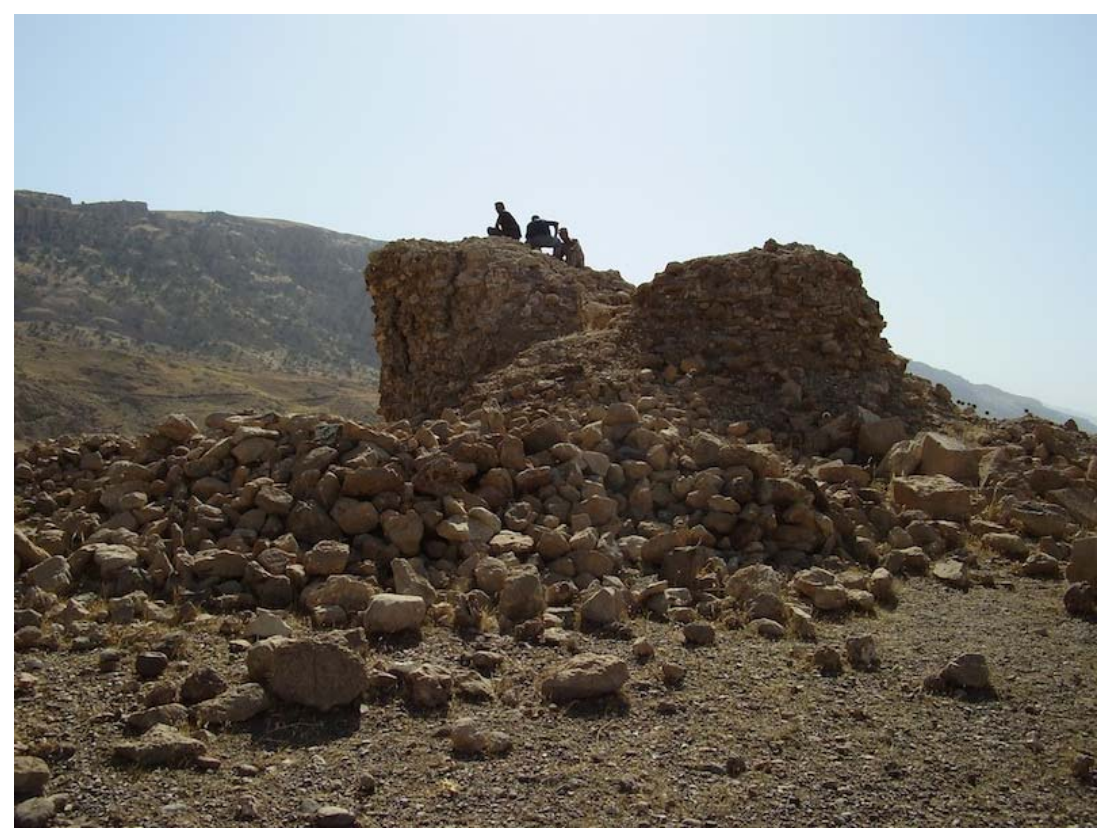

Fig. 2 - Paikuli, il monumento di Narseh (immagine MAIKI). 


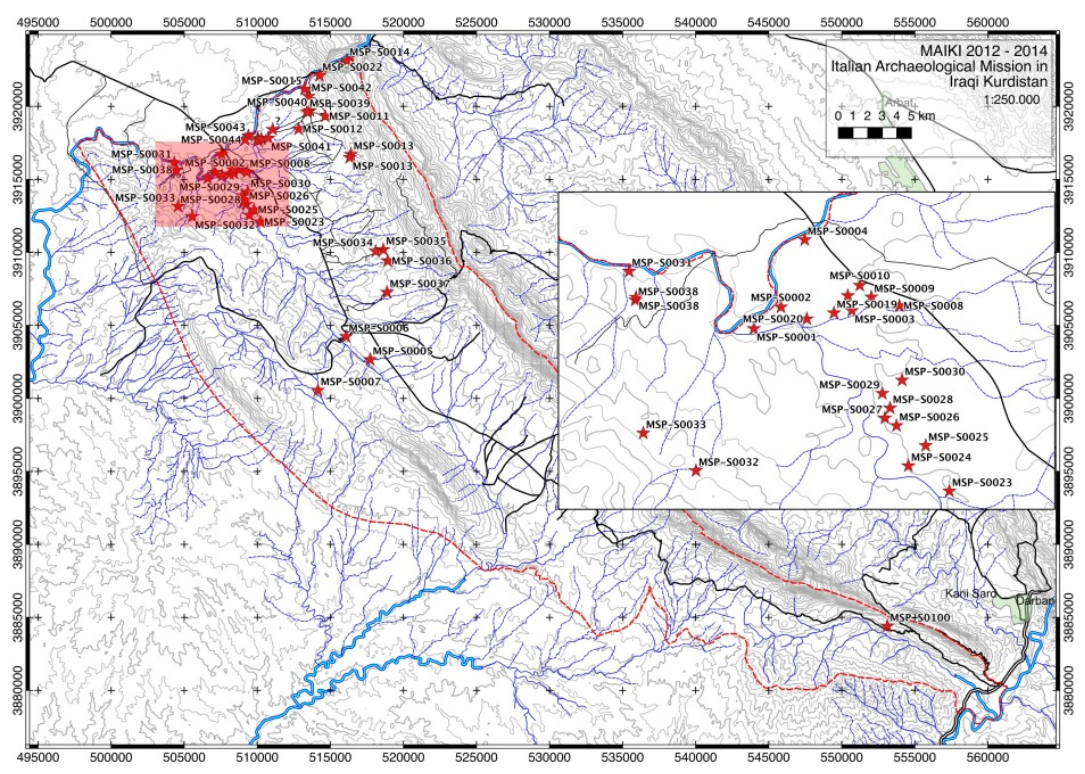

Fig. 3 - I siti storico-archeologici identificati dal MAIKI Survey Project (immagine MAIKI).

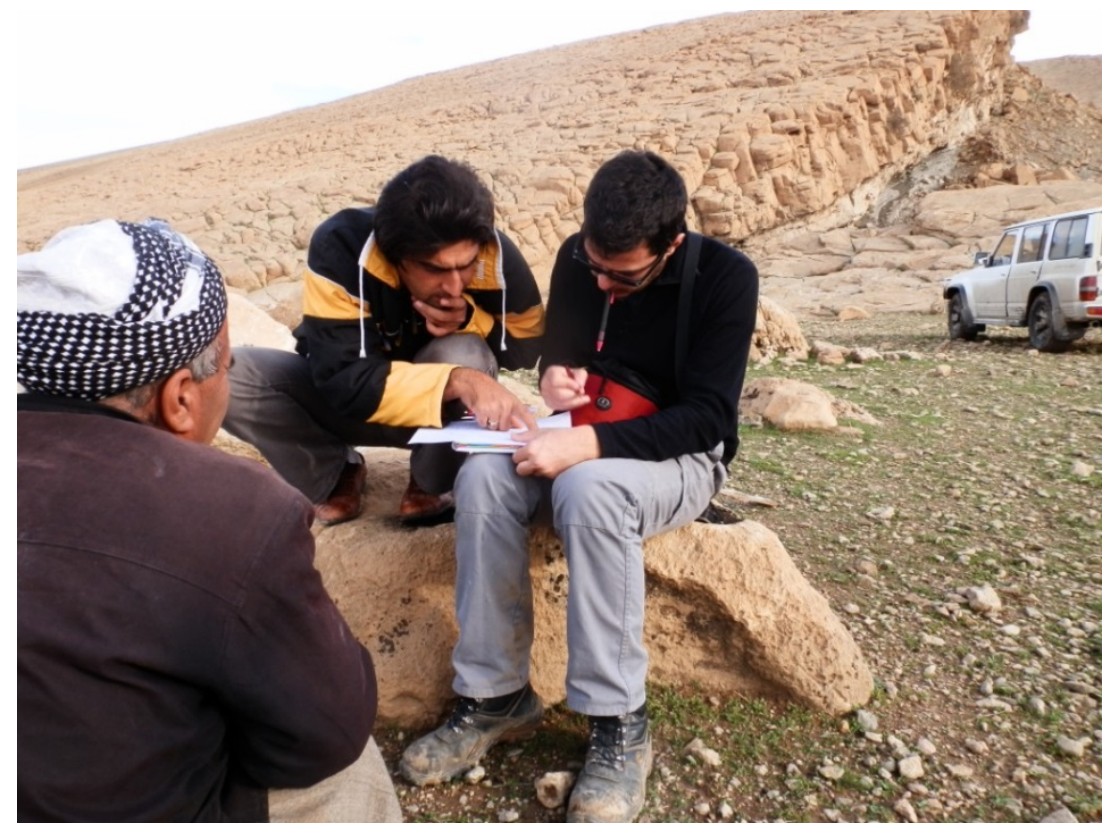

Fig. 4 - J. Bogdani e Nawshirwan Aziz Mohammed durante la survey (immagine MAIKI). 


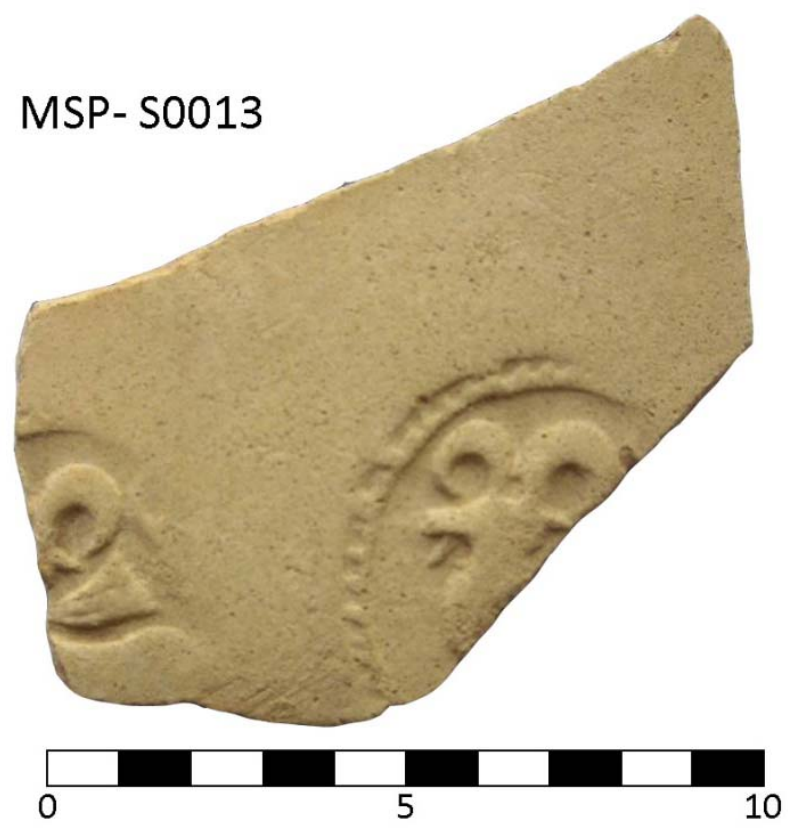

Fig. 5 - Ceramica non invetriata con decorazione a stampo in rilievo, epoca sasanide, sito MSP- S0013 (immagine MAIKI).

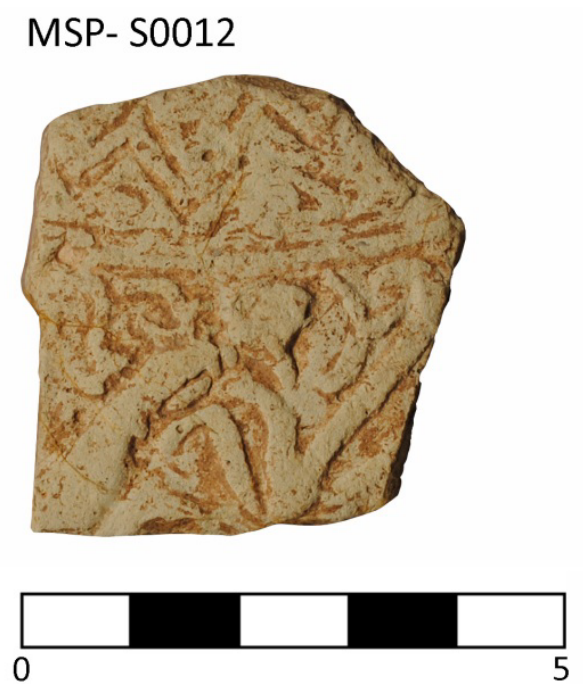

Fig. 6 - Ceramica non invetriata con decorazione a stampo in rilievo, epoca islamica, sito MSP-S0012 (immagine MAIKI). 


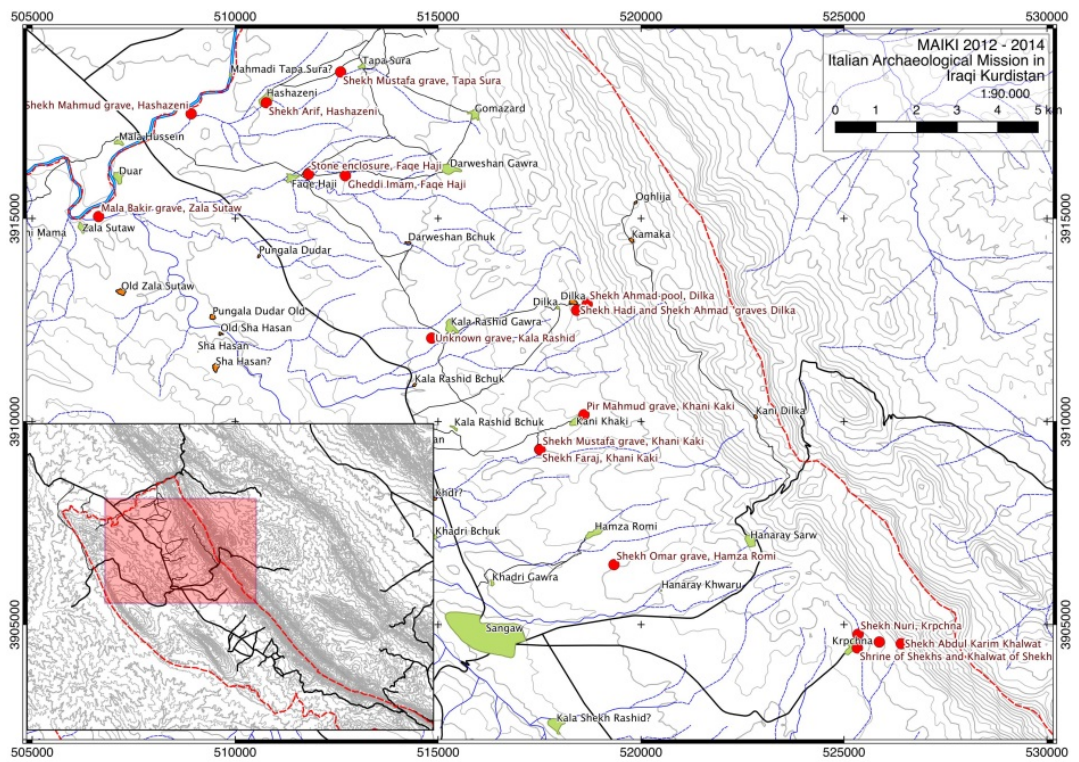

Fig. 7 - Luoghi di venerazione degli sheykh (immagine MAIKI).

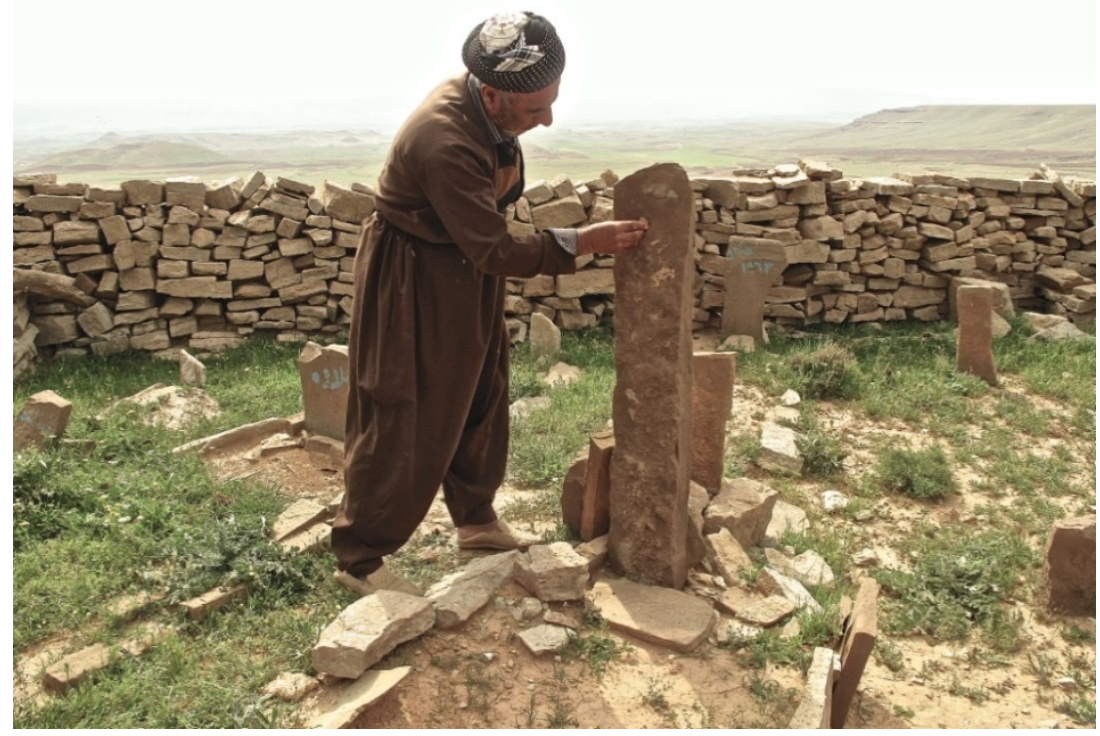

Fig. 8 - Cimitero di Dilka, tomba di Sheykh Aḥmad (immagine MAIKI). 